\title{
Incidences de la multiplication in vitro sur le comporte- ment ultérieur en culture de quelques variétés de gerbera
}

\author{
Jacques MEYNET \\ I.N.R.A., Station d'Amélioration des Plantes florales, La Gaudine, F 83600 Fréjus
}

\section{RÉSUMÉ}

\begin{abstract}
Plusieurs clones de gerbera ont été cultivés et multipliés in vitro selon la méthode de MAïA (1977). La comparaison entre boutures et vitroplants révèle de fortes interactions génotype $\times$ mode de multiplication. Ces effets différentiels de la vitroculture portent essentiellement sur la capacité des génotypes à recouvrer, après multiplication intensive in vitro, une dominance apicale forte et stable jusqu'à l'induction florale.

Parmi toutes les variétés observées, deux («Fredaisy " et "Fredimo ») s'avèrent différentes selon qu'elles proviennent de bouturage traditionnel ou de vitroculture et ces différences se maintiennent stables même après plusieurs cycles de multiplication clonale des vitroplants.

Cinq milieux différents de transition avant enracinement modifient sensiblement le comportement de toutes les variétés observées.

En revanche, l'origine du premier explant, qu'il provienne d'un méristème végétatif ou d'un bouton floral, parait sans effets sur le comportement variétal in vitro et en serre.
\end{abstract}

Mots clés additionnels : Gerbera jamesonii Bolus, culture in vitro, multiplication conforme, boutures, vitroplants.

Effects of in vitro propagation on the further growth behaviour of some Gerbera varieties.

Several gerbera clones have been cultivated and multiplied according to the MAïA method (1977). Comparison between cuttings and in vitro-plants showed high genotype $\times$ mode of multiplication interactions. In vitro culture mainly modified the genotype's ability to recover a strong and durable apical dominance after intensive in vitro multiplication. Among all the varieties observed, two ( Fredaisy » and « Fredimo») proved different according to their origin from traditional cuttings or from in vitro culture and these differences remained stable even after several clonal multiplication cycles of the in vitro plants. Five different transition media slightly modified the behaviour of all the observed varieties. In contrast, the origin of the first explant, whether from a vegetative meristem or a bud, seemed without effect on the in vitro and glasshouse behaviour of varieties.

Additional key words : Gerbera jamesonii Bolus, in vitro culture, true to type multiplication, cuttings, vitroplants.

\section{INTRODUCTION}

Il est possible aujourd'hui de multiplier in vitro le gerbera et d'utiliser pour la culture en serre les vitroplants ainsi obtenus. Les techniques de mise en culture, de multiplication et d'enracinement ont été décrites par plusieurs auteurs (Murashige et al., 1974 ; PIERIK, 1975 ; MAÏA, 1977), mais l'incidence de la multiplication in vitro de divers clones de gerbera sur leur comportement ultérieur en culture n'a jamais été décrite avec précision.

L'objet de ce présent travail fait un premier bilan sur ce problème après 4 années d'observations ou d'expérimentations sur le comportement, par rapport au bouturage naturel, des plantes cultivées en serre à partir des vitro- plants en fonction des variétés, des techniques de vitroculture et de l'origine de l'explant initial.

\section{MATÉRIEL ET MÉTHODES}

\section{A. Essai 1}

Six clones sélectionnés à Fréjus et non apparentés ont été reproduits soit par bouturage traditionnel, soit selon la technique de vitroculture mise au point par MAfa (1977) ; la mise en culture in vitro a été effectuée de 1976 à 1980, à partir de jeunes boutons floraux, ou de méristèmes végétatifs (tabl. 1). 
TABLEAU 1

Quelques caractéristiques des clones observés.

Some characteristics of the clones observed.

\begin{tabular}{|c|c|c|c|c|c|}
\hline \multicolumn{3}{|c|}{ Bouturage } & \multicolumn{3}{|c|}{ Vitroculture (E. Maïa) } \\
\hline & depuis & $\begin{array}{l}\text { Coeff. }\left({ }^{1}\right) \\
x\end{array}$ & année & $\begin{array}{l}\text { mise en culture } \\
\text { origine }\end{array}$ & $\underset{x}{\text { Coeff. }}\left({ }^{2}\right)$ \\
\hline Fredaisy & 1973 & 10 & 1976 & bouton floral & 3,5 \\
\hline Fredorella & 1975 & 10 & 1977 & bouton floral & 2,8 \\
\hline Fresamande & 1977 & 7 & $\begin{array}{l}1978 \\
1980\end{array}$ & $\begin{array}{l}\text { bouton floral }(A) \\
\text { méristème }(B)\end{array}$ & 2,7 \\
\hline Fresultane & 1976 & 7 & $\begin{array}{l}1980 \\
1980\end{array}$ & $\begin{array}{l}\text { bouton floral (A B C) } \\
\text { méristème (D) }\end{array}$ & 5,2 \\
\hline Fredeking & 1977 & 9 & 1979 & bouton floral & 2,8 \\
\hline Frescador & 1977 & 6 & 1979 & bouton floral & 3,7 \\
\hline $8175-1$ & 1978 & 8 & $\begin{array}{l}1980 \\
1980\end{array}$ & $\begin{array}{l}\text { méristème (A) } \\
\text { bouton floral (B C D) }\end{array}$ & 3,5 \\
\hline $8008-10$ & 1978 & 9 & 1980 & bouton floral (A B) & 2,5 \\
\hline Fredimo & 1975 & 8 & 1976 & bouton floral & 3,5 \\
\hline
\end{tabular}

Nous avons comparé le comportement en serre pour plusieurs variétés des plantes issues de vitroplants et de boutures traditionnelles.

Les notations caractérisant les comportements variétaux ont porté principalement sur le nombre des fleurs récoltées, soit pendant les 3 mois d'hiver (décembre + janvier + février), soit durant toute la campagne, et sur la qualité appréciée d'une façon subjective et synthétique et mesurée par le rapport du nombre de fleurs classées en $1^{\text {re }}$ catégorie sur le nombre total de fleurs récoltées. Nous avons parfois noté quelques éléments de la qualité florale (longueur de Ja tige, diamètre du capitule, poids), la précocité de floraison, la ramification des plants, et testé sur disques foliaires la sensibilité à Phytophthora cryptogea (ATHANASSIOU, 1979).

Les essais ont été mis en place en serre le 25 juin. Les vitroplants sortis de tubes le 30 avril étaient au stade $3^{\mathrm{e}}$ feuille néoformée ; les boutures au stade $2^{\mathrm{e}}$ feuille provenaient d'un prélèvement sur leurs pieds-mères effectué le 5 mai. Une expérience préliminaire nous avait montré qu'il était nécessaire de respecter ce calendrier pour obtenir un début de floraison à peu près synchrone survenant dans le courant du mojs de septembre, quel que soit le mode de multiplication.

Deux essais clisposés en split-plot (avec les variétés pour objet principal et les modes de multiplication pour objet secondaire) ont été suivis successivement en 1980-81 (essai à 3 répétitions), puis 1981-82 (4 répétitions). Par ailleurs, pour juger de l'éventuelle stabilité des variations observées, des boutures prélevées sur les vitroplants de la culture 198081 ont été ajoutées au dispositif expérimental. Les objectifs étant distincts, nous analyserons séparément les couples boutures-vitroplants et les couples boutures de bouturesboutures de vitroplants.

Enfin, pour 5 autres génotypes, 10 boutures ont été comparées à 10 vitroplants.

Les essais ont été réalisés dans une serre conduite aux températures minimales de $18^{\circ} \mathrm{C}$ dans le sol et $10^{\circ} \mathrm{C}$ dans l'air. Dans tous les dispositifs, analysés statistiquement, chaque parcelle élémentaire était constituée de 5 plants.

\section{B. Essai 2}

Dans un $2^{\mathrm{e}}$ essai, nous avons comparé, selon le même dispositif expérimental que précédemment (4 répétitions, variétés pour objet principal ; vitrotechniques pour objet secondaire), des vitroplants obtenus par divers modes de multiplication.

Les clones ont été repiqués régulièrement toutes les 4 semaines sur le milieu de prolifération de MAÏA. Quatre semaines avant le transfert sur le milieu d'enracinement commun (le 10 mars 1981), s'intercale un seul cycle différentiel de transition; les explants sont repiqués sur différents milieux, les 3 premiers réalisant un gradient croissant de cytokinine :

I. La concentration en cytokinine est réduite de moitié,

II. Milieu témoin de MAÏA,

III. La concentration en cytokinine est doubléc,

IV. Auxines et cytokinines sont également réduites 5 fois, $\mathrm{V}$. Les explants sont maintenus sans repiquage sur le milieu témoin pendant 8 semaines.

\section{Essai 3}

Dans un $3^{\mathrm{e}}$ essai, nous avons étudié l'influence de l'origine du $1^{\text {er }}$ explant sur le comportement des vitroplants. Les vitroplants de plusieurs cultivars proviennent soit de boutons floraux distincts, soit de méristèmes axillaires végétatifs. Ayant maintenu séparées ces différentes origines, nous avons pu comparer différentes souches :

$\begin{array}{cc}\text { provenant } & \text { provenant } \\ \text { de boutons floraux } & \text { de méristèmes }\end{array}$

$\begin{array}{lll}\text { Frésultane } & \text { A B C } & \text { D } \\ 8175-1 & \text { B C D } & \text { A } \\ 8008-10 & \text { A B } & \\ \text { Frésamande } & \text { A } & \text { B }\end{array}$

Nous avons observé attentivement des caractères morphologiques et noté les productions florales de 2 répétitions de 5 plants de chaque souche. 


\section{RÉSULTATS}

A. Comparaison de deux techniques de multiplication, bouturage et vitroculture, sur le comportement en culture de divers clones

1. Essai de 6 variêtés multipliées par bouturage ou par vitroculture

Le tableau 2 d'analyse de variance révèle en particulier une forte interaction variété $\times$ mode de multiplication, ce qui nécessite une étude variété par variété. En première analyse, la vitrométhode MAÏA peut, selon les cultivars, induire une baisse ("Fredaisy»), une stabilité ("Fredeking ») ou une augmentation ( «Frescador $»$ ) du nombre de fleurs produites (tabl. 3). Toutefois dans tous les cas, sauf pour "Fresultane ", la qualité florale est affectée négativement. Cette dégradation, d'intensité très variable selon les génotypes, se manifeste davantage par une réduction du diamètre, surtout du poids total et par un défaut de lignification que par une modification de la longueur des tiges (tabl. 4).

Une étude des productions florales durant les 3 mois d'hiver (décembre + janvier + février) aboutit à des résultats comparables; seule la qualité est davantage affectée ; les boutures donnent en général des fleurs de qualité légèrement supérieure alors que les vitroplants sont assez nettement moins bons durant cette période que tout au cours de la campagne entière.

\section{Observations de 5 autres génotypes (tabl. 5)}

Ces résultats confirment la grande spécificité des réactions variétales à la vitroculture. Si l'on considère l'ensemble des 11 génotypes observés nous constatons une corrélation significative entre :

- La précocité de floraison des vitroplants et des boutures, $r=+0,65\left(^{*}\right)$.

- Le taux de prolifération in vitro et la ramification des boutures notée en janvier, $r=+0,71\left(^{*}\right)$.

\section{TABLEAU 2}

Analyse de variances des productions totales jusqu'à fin avril toutes qualités confondues. Analysis of variance of total production up to late April.

\begin{tabular}{|c|c|c|c|c|c|c|c|c|}
\hline & \multicolumn{4}{|c|}{$1980-81$} & \multicolumn{4}{|c|}{$1981-82$} \\
\hline & $\begin{array}{c}\text { Somme } \\
\text { des carrés }\end{array}$ & ddl & $\begin{array}{l}\text { Carrés } \\
\text { moyens }\end{array}$ & $\mathrm{F}$ & $\begin{array}{c}\text { Somme } \\
\text { des carrés }\end{array}$ & ddl & $\begin{array}{l}\text { Carrés } \\
\text { moyens }\end{array}$ & $\mathrm{F}$ \\
\hline Blocs & 439,5 & 2 & 246,7 & 1,19 & 458,0 & 3 & 152,7 & 1,41 \\
\hline Variétés (V) & 4088,8 & 5 & 817,7 & $3,96\left(^{*}\right)$ & 8492,2 & 5 & 1698,4 & $15,73(* *)$ \\
\hline Erreur (a) & 2066,2 & 10 & 206,6 & & 1620,5 & 15 & 108,0 & \\
\hline Total (a) & 6648,5 & 17 & & & 10570,7 & 23 & & \\
\hline Mode de x (M) & 890,4 & 1 & 890,4 & 3,43 & 1050,8 & 1 & 1050,8 & $11,6(* *)$ \\
\hline Interaction $\mathrm{V} \times \mathrm{M}$ & 5690,5 & 5 & 1138,1 & $7,14(* *)$ & 2628,2 & 5 & 531,6 & $5,87(* *)$ \\
\hline Erreur (b) & 1912,8 & 12 & 159,4 & & 1630,0 & 18 & 90,56 & \\
\hline Total & 15142,2 & 35 & & & 15879,7 & 47 & & \\
\hline
\end{tabular}

(*) F significatif au seuil 0,05 .

(**) F significatif au seuil 0,01 .

TABLEAU 3

Nombre de fleurs par plante et qualité moyenne des fleurs récoltées jusqu'à la fin avril. Number of flowers per plant and mean quality of yields up to late April.

\begin{tabular}{|c|c|c|c|c|c|}
\hline \multirow[t]{2}{*}{ Variétés } & & \multicolumn{2}{|c|}{ Nombre } & \multicolumn{2}{|c|}{ Qualité ( $\left.{ }^{1}\right)$} \\
\hline & & Boutures & Vitro & Boutures & Vitro \\
\hline Fredaisy & $\begin{array}{l}80-81 \\
81-82\end{array}$ & $\begin{array}{l}17,7 \\
21,2\end{array}$ & $\begin{array}{l}14,2\left(^{*}\right) \\
17,6\left(^{* *}\right)\end{array}$ & $\begin{array}{l}56,0 \\
56,6\end{array}$ & $\begin{array}{l}27,3 \\
30,7\end{array}$ \\
\hline Fredorella & $\begin{array}{l}80-81 \\
81-82\end{array}$ & $\begin{array}{l}10,8 \\
17,9\end{array}$ & $\begin{array}{l}14,1\left(^{*}\right) \\
20,3\left(^{*}\right)\end{array}$ & $\begin{array}{l}70,4 \\
71,3\end{array}$ & $\begin{array}{l}82,3 \\
87,5\end{array}$ \\
\hline Fresamande $\mathrm{A}$ & $\begin{array}{l}80-81 \\
81-82\end{array}$ & $\begin{array}{l}12,1 \\
13,0\end{array}$ & $\begin{array}{l}16,5\left(^{* *}\right) \\
16,6\left(^{* *}\right)\end{array}$ & $\begin{array}{l}94,3 \\
87,0\end{array}$ & $\begin{array}{l}79,5 \\
59,7\end{array}$ \\
\hline Fresultane C & $\begin{array}{l}80-81 \\
81-82\end{array}$ & $\begin{array}{l}13,1 \\
10,4\end{array}$ & $\begin{array}{l}14,6 \\
15,6\left(^{* *}\right)\end{array}$ & $\begin{array}{l}88,2 \\
83,3\end{array}$ & $\begin{array}{l}87,4 \\
81,3\end{array}$ \\
\hline Fredeking & $\begin{array}{l}80-81 \\
81-82\end{array}$ & $\begin{array}{l}10,3 \\
11,2\end{array}$ & $\begin{array}{l}12,1 \\
13,2\end{array}$ & $\begin{array}{l}73,5 \\
56,7\end{array}$ & $\begin{array}{l}61,5 \\
55,7\end{array}$ \\
\hline Frescador & $\begin{array}{l}80-81 \\
81-82\end{array}$ & $\begin{array}{r}9,8 \\
13,6\end{array}$ & $\begin{array}{l}14,1\left(^{* *}\right) \\
18,1\left(^{* *}\right)\end{array}$ & $\begin{array}{l}95,8 \\
88,7\end{array}$ & $\begin{array}{l}89,8 \\
73,3\end{array}$ \\
\hline
\end{tabular}

$\left.{ }^{1}\right)$ Qualité $=($ nombre de fleurs en catégorie $\mathrm{I} /$ nombre total $) \times 100$.

$\left({ }^{*}\right)=$ différence entre vitro et bouture significative au seuil 0,05 .

$\left({ }^{* *}\right)=$ différence entre vitro et bouture significative au seuil 0,01 . 
TABLEAU 4

Quelques caractéristiques des 6 clones multipliés par bouturage (B) et par vitroculture (V) testés en 1980-81. Some characteristics of 6 clones multiplied by cuttings $(B)$ or by in vitro culture $(V)$ and tested in 1980-81.

\begin{tabular}{|c|c|c|c|c|c|c|c|c|c|c|}
\hline & \multicolumn{2}{|c|}{ Ramification } & \multicolumn{2}{|c|}{ Longueur } & \multicolumn{2}{|c|}{ Largeur } & \multicolumn{2}{|c|}{ Poids frais } & \multicolumn{2}{|c|}{ Nécrose phyto } \\
\hline & B & V & B & $\mathrm{V}$ & B & V & B & V & B & $\mathrm{V}$ \\
\hline Fredaisy & 1,3 & 1,5 & 63,5 & 66,6 & 11,5 & $10,6\left(^{*}\right)$ & 23,3 & $18,4\left(^{*}\right)$ & 28,4 & 28,8 \\
\hline Fredorella & 1,0 & $1,8(* *)$ & 58,1 & 56,3 & 10,5 & 10,6 & 24,1 & 23,9 & 25,3 & 25,8 \\
\hline Fresamande A & 1,1 & $2,7(* *)$ & 62,7 & 60,5 & 10,4 & 10,1 & 33,5 & $28,2(* *)$ & 13,1 & 12,9 \\
\hline Fresultane C & 1,2 & $2,1(*)$ & 60,5 & 61,1 & 11,1 & 11,0 & 32,3 & 29,4 & 25,7 & 25,4 \\
\hline Fredeking & 1.0 & $1,8\left(^{* *}\right)$ & 49,7 & 48,7 & 11,3 & 10,9 & 25,2 & $22,3\left(^{*}\right)$ & 27,8 & 26,1 \\
\hline Frescador & 1,2 & $2,2(* *)$ & 65,0 & 64,2 & 11,5 & 11,4 & 34,1 & 33,5 & 28,3 & 29,5 \\
\hline Moyenne & 1,1 & $2,0(* *)$ & 59,9 & 59,6 & 11,1 & $10,8\left(^{*}\right)$ & 28,8 & $20,4\left(^{* *}\right)$ & 24,8 & 24,7 \\
\hline
\end{tabular}

Ramification : rombre de méristèmes actifs simultanément 8 semaines après plantation.

Branching : number of simultaneously active meristems 8 weeks after planting.

Longueur: de la tige en $\mathrm{cm}$.

Length of the stem.

Largeur : du capitule en mm.

Diameter of the capitulum.

Poids frais : de la fleur entière en $g$.

Fresh weight of the whole flower.

Nécrose phyto. : diamètre de nécrose provoquée par un implant de Phytophthora cryptogea sur disque foliaire.

Diameter of the necrosis induced by a Phytophthora cryptogea implant on a foliar disc.

B : boutures.

cuttings

$\mathrm{V}:$ vitro plants.

\section{TABLEAU 5}

Valeurs individuelles de 5 clones multipliés par bouturage ou par vitroculture (moyennes de 10 plantes) en 1980-81. Individual values of 5 clones multiplied by cutting or by in vitro culture (means of 10 plants) in 1980-81.

\begin{tabular}{|c|c|c|c|c|c|c|}
\hline \multirow[t]{2}{*}{ Génotypes } & & \multirow[t]{2}{*}{ Ramification } & \multicolumn{2}{|c|}{ Production hivernale } & \multicolumn{2}{|c|}{ Production totale } \\
\hline & & & Nombre & Qualité & Nombre & Qualité \\
\hline $9018-8$ & $\begin{array}{l}\text { boutures } \\
\text { vitro } \\
\text { écart \% }\end{array}$ & $\begin{array}{l}1,0 \\
1,2\end{array}$ & $\begin{array}{r}4,6 \\
4,9 \\
+\quad 6,5\end{array}$ & $\begin{array}{l}95,7 \\
93,9\end{array}$ & $\begin{array}{r}14,2 \\
16,2 \\
+14,1\end{array}$ & $\begin{array}{l}93,0 \\
92,0\end{array}$ \\
\hline $8230-10$ & $\begin{array}{l}\text { boutures } \\
\text { vitro } \\
\text { écart \% }\end{array}$ & $\begin{array}{l}1,0 \\
1,1\end{array}$ & $\begin{array}{r}3,2 \\
5,1 \\
+59,4\end{array}$ & $\begin{array}{l}72,6 \\
80,4\end{array}$ & $\begin{array}{r}12,6 \\
15,2 \\
+20,6\end{array}$ & $\begin{array}{l}81,0 \\
81,5\end{array}$ \\
\hline $8089-1$ & $\begin{array}{l}\text { boutures } \\
\text { vitro } \\
\text { écart \% }\end{array}$ & $\begin{array}{l}1,0 \\
1,4\end{array}$ & $\begin{array}{r}5,0 \\
6,0 \\
+20,0\end{array}$ & $\begin{array}{l}80,0 \\
75,0\end{array}$ & $\begin{array}{r}16,8 \\
20,1 \\
+19,6\end{array}$ & $\begin{array}{l}82,1 \\
80,6\end{array}$ \\
\hline $9281-9$ & $\begin{array}{l}\text { boutures } \\
\text { vitro } \\
\text { écart \% }\end{array}$ & $\begin{array}{l}1,4 \\
2,9\end{array}$ & $\begin{array}{r}4,3 \\
4,8 \\
+11,6\end{array}$ & $\begin{array}{l}81,5 \\
60,4\end{array}$ & $\begin{array}{r}13,9 \\
15,7 \\
+12,9\end{array}$ & $\begin{array}{l}82,3 \\
59,2\end{array}$ \\
\hline Fredimo & $\begin{array}{l}\text { boutures } \\
\text { vitro } \\
\text { écart \% } \\
\text { boutures de vitro }\end{array}$ & $\begin{array}{l}1,0 \\
3,8 \\
1,0\end{array}$ & $\begin{array}{r}4,5 \\
1,4 \\
-68,9 \\
2,8\end{array}$ & $\begin{array}{l}76,8 \\
40,2 \\
48,2\end{array}$ & $\begin{array}{r}15,7 \\
8,9 \\
-43,3 \\
10,4\end{array}$ & $\begin{array}{l}83,5 \\
53,1 \\
49,4\end{array}$ \\
\hline
\end{tabular}

- Le taux de prolifération in vitro et la tardiveté des vitroplants, $\mathrm{r}=+0,91\left({ }^{* *}\right)$.

Ces corrélations suggèrent un mécanisme physiologique de la prolifération végétative dépendant essentiellement des cytokinines endogènes ou exogènes.

\section{B. Stabilité de la variation induite par vitroculture}

Selon les variétés, l'utilisation des vitroplants peut, comparativement aux boutures traditionnelles, modifier le comportement des plantes qui en sont issues. Pour vérifier si ces modifications induites sont transitoires ou se maintien- nent au cours des générations clonales successives, nous avons comparé 6 clones reproduits par bouturage à partir soit de boutures soit de vitroplants. L'analyse de variance des productions hivernales et totales indique une interaction variété $\times$ origine des boutures hautement significative, nous nous contenterons donc de reporter les performances par variétés dans le tableau 6. Il apparaît que seule la variété "Fredaisy " ne s'avère plus conforme au témoin bouturé de façon traditionnelle depuis 8 ans et que, dans ce seul cas, les défauts décelés sur vitroplants se maintiennent après bouturage.

Remarque 1: Nous devons noter par ailleurs que les 
TABLEAU 6

Productions individuelles moyennes de 6 clones bouturés à partir soit de bouture $(B B)$, soit de vitroplant $(B V)$. Individual mean yields of 6 clones propagated by cuttings whether from cuttings $(B B)$ or from in vitro plants (BV).

\begin{tabular}{|c|c|c|c|c|c|c|c|c|}
\hline & \multicolumn{4}{|c|}{ Production hivernale } & \multicolumn{4}{|c|}{ Production totale } \\
\hline & \multicolumn{2}{|c|}{ Nombre } & \multicolumn{2}{|c|}{ Qualité } & \multicolumn{2}{|c|}{ Nombre } & \multicolumn{2}{|c|}{ Qualité } \\
\hline & $\mathrm{BB}$ & BV & $\mathrm{BB}$ & BV & $\mathrm{BB}$ & $\mathrm{BV}$ & $\mathrm{BB}$ & BV \\
\hline Fredaisy & 8,0 & $5,3\left(^{*}\right)$ & 61,0 & 39,8 & 21,2 & $14,2(* *)$ & 56,6 & 35,8 \\
\hline Fredorella & 6,1 & 5,8 & 60,5 & 65,7 & 17,9 & 19,1 & 71,3 & 72,3 \\
\hline Fresamande & 4,6 & 4,3 & 90,9 & 88,2 & 13,0 & 12,4 & 87,0 & 92,1 \\
\hline Fresultane & 3,8 & 3,5 & 81,5 & 78,2 & 10,4 & 10,8 & 83,3 & 86,8 \\
\hline Fredeking & 4,7 & 5,1 & 63,6 & 56,0 & 11,2 & 12,1 & 56,7 & 55,7 \\
\hline Frescador & 4,5 & 3,9 & 95,0 & 87,3 & 13,6 & 13,9 & 88,7 & 80,0 \\
\hline
\end{tabular}

vitroplants ont fourni en moyenne davantage de boutures $(10,2)$ que les pieds-mères provenant eux-mêmes de boutures $(8,3$ boutures/pieds-mères).

Remarque 2: Les boutures prélevées sur les vitroplants de «Fredimo» conservent également les défauts de production de leurs plantes-mères (tabl. 5 , observations sur 10 plants sans répétition).

\section{Incidence de diverses techniques de vitroculture sur le comportement variétal}

Le tableau 7 indique les effets immédiats de la vitroculture ; toute modification subite (même une teneur accrue en cytokinine) au régime routinier de prolifération (formule II) provoque, dans un premier temps, une chute du taux de prolifération; au gradient croissant de cytokinine correspond un allongement des délais d'enracinement et une diminution du nombre de racines, ces dernières données étant inversement corrélées $(r=0,84)$.

$\mathrm{La}$ ramification précoce des plantes obtenues après vitroculture et leur précocité de floraison (tabl. 8) ainsi que leurs productions hivernale et totale (tabl. 9) dépendent largement des génotypes mais aussi de la nature du milieu de transition avant enracinement. Cependant pour chacun de ces paramètres aucune interaction génotype $\times$ vitrotechnique n'a pu être mise en évidence.

\section{TABLEAU 7}

Incidences de 5 formules de transition avant enracinement sur le taux moyen de multiplication, le nombre moyen de racines après 21 jours d'enracinement, la vitesse d'enracinement mesurée en nombre de jours écoulés du transfert sur milieu d'enracinement à l'émission des premières ébauches racinaires.

Effects of 5 transition media before rooting on: the mean multiplication coefficient, the mean number of roots after 21 days of rooting, the rooting rapidity measured in days from the transferring on the rooting medium until the emergence of the first roots.

\begin{tabular}{lccc}
\hline \hline Formules & $\begin{array}{c}\text { Taux de } \\
\text { multiplication }\end{array}$ & $\begin{array}{c}\text { Nombre } \\
\text { de racines }\end{array}$ & $\begin{array}{c}\text { Vitesse } \\
\text { d'enracinement }\end{array}$ \\
\hline I & 2,97 & 3,12 & 6,7 \\
II & 3,15 & 3,02 & 8,0 \\
III & 2,01 & 2,50 & 8,8 \\
IV & 1,69 & 2,90 & 7,0 \\
V & $4,73\left({ }^{1}\right)$ & 3,23 & 6,8 \\
\hline
\end{tabular}

( ${ }^{1}$ Après 8 semaines au lieu de 4 pour les autres formules.

Nous noterons en particulier que la multiplication routinière sans transition (traitement II) stimule la ramification, retarde la floraison et affecte la qualité de la floraison

\section{TABLEAU 8}

Analyse des ramifications précoces (notations 5 semaines après plantation du nombre de méristèmes actifs simultanément) et des précocités de floraison de 6 variétés conduites in vitro de 5 façons différentes.

Analysis of early branching (scored 5 weeks after planting) and of flowering earliness in 6 varieties cultivated in vitro in 5 different ways.

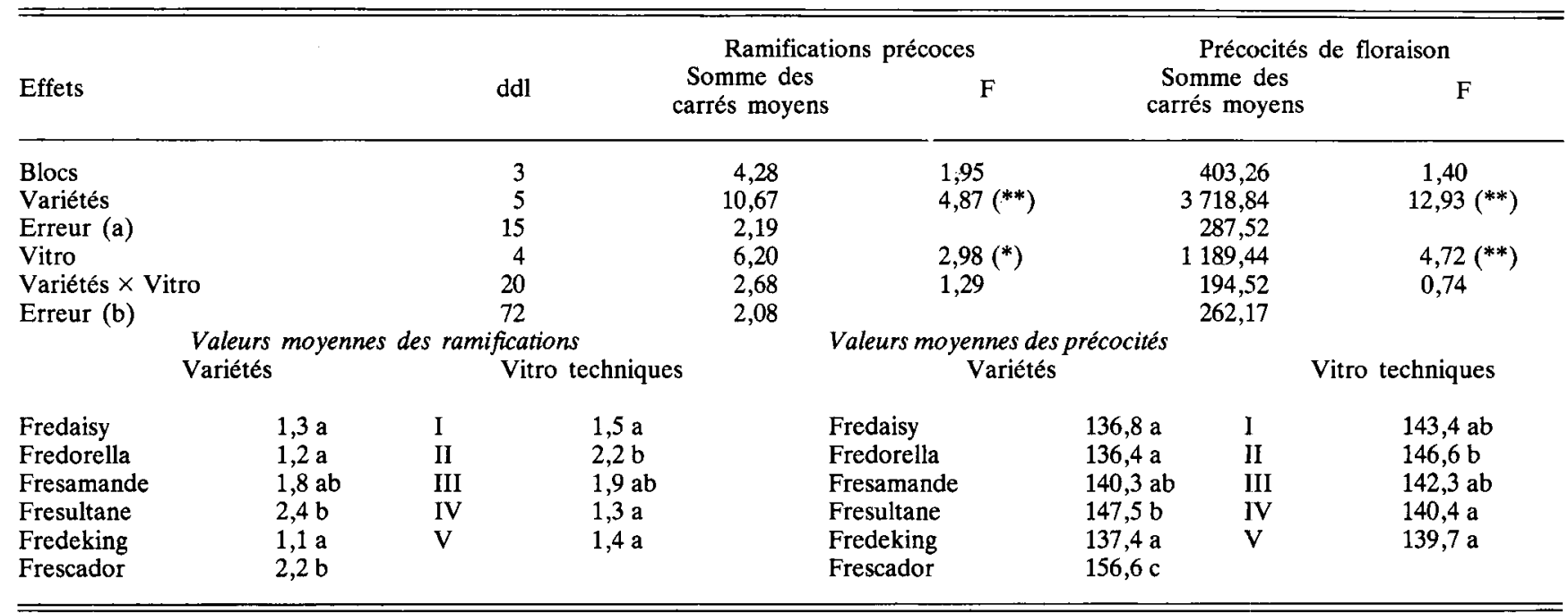


TABLEAU 9

Analyse des productions hivernales et totales de 6 variétés multipliées in vitro selon 5 techniques différentes.

Analysis of winter and total yields of 6 varieties cultivated in vitro in 5 differents ways.

\begin{tabular}{|c|c|c|c|c|c|}
\hline Effets & $\mathrm{ddl}$ & $\begin{array}{l}\text { Productio } \\
\text { Somme des } \\
\text { carrés moyens }\end{array}$ & nale & $\begin{array}{l}\text { Product } \\
\text { Somme des } \\
\text { carrés moyens }\end{array}$ & $\mathrm{F}$ \\
\hline Blocs & 3 & 15,90 & 0,66 & 47,99 & 0,51 \\
\hline Variétés & 5 & 337,34 & $13,92(* *)$ & 1833,19 & $19,37(* *)$ \\
\hline Erreur (a) & 15 & 24,24 & & 94,66 & \\
\hline Vitro & 4 & 91,47 & $3,99\left(^{*}\right)$ & 413,51 & $3,32\left(^{*}\right)$ \\
\hline Variétés $\times$ vitro & 20 & 63,29 & 2,76 & 291,96 & 2,34 \\
\hline Erreur (b) & 72 & 22,93 & & 124,67 & \\
\hline
\end{tabular}

Moyennes individuelles

Production hivernale

Production totale

Vitro techniques

$\begin{array}{llr}\text { I } & 5,55 \mathrm{a} & 16,27 \mathrm{a} \\ \text { II } & 6,09 \mathrm{ab} & 16,40 \mathrm{a} \\ \text { III } & 5,86 \mathrm{a} & 16,82 \mathrm{a} \\ \text { IV } & 5,66 \mathrm{a} & 16,48 \mathrm{a} \\ \text { V } & 6,53 \mathrm{~b} & 18,29 \mathrm{~b}\end{array}$

TABLEAU 10

Qualité florale moyenne de 6 variétés après culture in vitro sur 5 milieux de transition différents.

Mean flower quality of 6 varieties after in vitro culture on 5 different transition media.

\begin{tabular}{|c|c|c|c|c|c|c|c|}
\hline \multirow{2}{*}{$\begin{array}{l}\text { Milieux } \\
\text { Variétés }\end{array}$} & & \multirow{2}{*}{ I } & \multirow{2}{*}{ II } & \multirow{2}{*}{ III } & \multirow{2}{*}{ IV } & \multirow{2}{*}{$\mathrm{V}$} & \multirow{2}{*}{$\frac{\text { Total I }}{\text { Total général }}$} \\
\hline & & & & & & & \\
\hline Fredaisy & $\begin{array}{l}\text { Hiver } \\
\text { Total }\end{array}$ & $\begin{array}{l}38,8 \\
26,9\end{array}$ & $\begin{array}{l}35,8 \\
28,3\end{array}$ & $\begin{array}{l}25,8 \\
24,6\end{array}$ & $\begin{array}{l}37,6 \\
28,6\end{array}$ & $\begin{array}{l}39,2 \\
35,7\end{array}$ & $\begin{array}{l}35,1 \\
28,8\end{array}$ \\
\hline Fredorella & $\begin{array}{l}\text { Hiver } \\
\text { Total }\end{array}$ & $\begin{array}{l}78,9 \\
87,2\end{array}$ & $\begin{array}{l}80,0 \\
87,5\end{array}$ & $\begin{array}{l}72,7 \\
82,2\end{array}$ & $\begin{array}{l}85,7 \\
85,1\end{array}$ & $\begin{array}{l}71,8 \\
77,5\end{array}$ & $\begin{array}{l}77,8 \\
84,0\end{array}$ \\
\hline Fresamande & $\begin{array}{l}\text { Hiver } \\
\text { Total }\end{array}$ & $\begin{array}{l}82,6 \\
73,2\end{array}$ & $\begin{array}{l}61,8 \\
59,7\end{array}$ & $\begin{array}{l}61,8 \\
72,9\end{array}$ & $\begin{array}{l}72,3 \\
77,6\end{array}$ & $\begin{array}{l}91,4 \\
84,5\end{array}$ & $\begin{array}{l}74,5 \\
74,4\end{array}$ \\
\hline Fresultane & $\begin{array}{l}\text { Hiver } \\
\text { Total }\end{array}$ & $\begin{array}{l}80,4 \\
86,0\end{array}$ & $\begin{array}{l}72,9 \\
77,2\end{array}$ & $\begin{array}{l}65,5 \\
76,8\end{array}$ & $\begin{array}{l}81,0 \\
90,8\end{array}$ & $\begin{array}{l}92,0 \\
93,2\end{array}$ & $\begin{array}{l}78,8 \\
85,2\end{array}$ \\
\hline Fredeking & $\begin{array}{l}\text { Hiver } \\
\text { Total }\end{array}$ & $\begin{array}{l}43,5 \\
47,9\end{array}$ & $\begin{array}{l}43,5 \\
49,1\end{array}$ & $\begin{array}{l}46,4 \\
44,7\end{array}$ & $\begin{array}{l}61,8 \\
50,0\end{array}$ & $\begin{array}{l}56,0 \\
56,7\end{array}$ & $\begin{array}{l}51,1 \\
50,1\end{array}$ \\
\hline Frescador & $\begin{array}{l}\text { Hiver } \\
\text { Total }\end{array}$ & $\begin{array}{l}87,5 \\
90,4\end{array}$ & $\begin{array}{l}62,3 \\
78,7\end{array}$ & $\begin{array}{l}63,3 \\
82,9\end{array}$ & $\begin{array}{l}90,3 \\
90,5\end{array}$ & $\begin{array}{l}71,5 \\
80,6\end{array}$ & $\begin{array}{l}74,3 \\
84,4\end{array}$ \\
\hline Total I & Hiver & 69,3 & 62,8 & 61,5 & 71,2 & 70,7 & \\
\hline Total général & Total & 69,5 & 65,1 & 63,8 & 73,4 & 71,5 & \\
\hline
\end{tabular}

(tabl. 10) ; en revanche, le maintien prolongé de 8 semaines dans ce même milieu de prolifération (traitement $\mathrm{V}$ ) apparaît le meilleur tout en produisant un nombre supérieur de fleurs.

\section{Influence de l'origine de l'explant initial}

Plusieurs souches ont été obtenues à partir de boutons floraux distincts chez 4 variétés et, en outre, pour 3 d'entre elles, de méristèmes végétatifs. Les 2 répétitions mises en place ne permettent de constater aucune différence entre les origines de vitroplants (tabl. 11).

\section{DISCUSSION}

Cette étude montre que la multiplication in vitro adéquate de certains cultivars peut apporter, outre les garanties sanitaires évidentes, une augmentation significative de la floribondité sans altération sensible de la qualité. Ce résultat positif et très prometteur est certainement à rapprocher de celui déjà obtenu en comparant boutures et plants issus de graines (DuClos \& MEYNET, 1977), le comportement de vitroplants pouvant être plus ou moins assimilé à celui de gerbera de semis. 


\section{TABLEAU 11}

Caractéristiques de production de plusieurs souches de vitroplants initiés à partir de boutons floraux ou de méristèmes ( $\left.{ }^{l}\right)$.

Production characteristics of several in vitro plants initiated from buds or meristems $\left({ }^{l}\right)$.

\begin{tabular}{|c|c|c|c|c|c|}
\hline & & \multicolumn{2}{|c|}{ Production hivernale } & \multicolumn{2}{|c|}{ Production totale } \\
\hline & & Nombre & Qualité & Nombre & Qualité \\
\hline Fresultane & $\begin{array}{l}\text { A } \\
\text { B } \\
\text { C } \\
\text { D }\left(^{1}\right)\end{array}$ & $\begin{array}{l}6,3 \\
5,9 \\
6,0 \\
5,9\end{array}$ & $\begin{array}{l}85,7 \\
81,4 \\
89,9 \\
91,5\end{array}$ & $\begin{array}{l}17,3 \\
17,1 \\
14,6 \\
16,7\end{array}$ & $\begin{array}{l}87,9 \\
81,3 \\
89,7 \\
94,5\end{array}$ \\
\hline 8175.1 & $\begin{array}{l}A\left({ }^{1}\right) \\
B \\
C \\
D\end{array}$ & $\begin{array}{l}5,9 \\
6,3 \\
5,8 \\
5,4\end{array}$ & $\begin{array}{l}96,8 \\
96,8 \\
93,1 \\
96,3\end{array}$ & $\begin{array}{l}15,8 \\
18,0 \\
15,9 \\
15,8\end{array}$ & $\begin{array}{l}95,6 \\
91,6 \\
88,0 \\
88,7\end{array}$ \\
\hline 8008.10 & $\begin{array}{l}\mathrm{A} \\
\mathrm{B}\end{array}$ & $\begin{array}{l}7,9 \\
8,7\end{array}$ & $\begin{array}{l}63,3 \\
69,1\end{array}$ & $\begin{array}{l}21,0 \\
23,0\end{array}$ & $\begin{array}{l}60,9 \\
63,3\end{array}$ \\
\hline Fresamande & $\begin{array}{l}\mathrm{A} \\
\mathrm{B}\left({ }^{1}\right)\end{array}$ & $\begin{array}{l}5,1 \\
4,9\end{array}$ & $\begin{array}{l}63,2 \\
65,1\end{array}$ & $\begin{array}{l}16,4 \\
16,2\end{array}$ & $\begin{array}{l}64,3 \\
64,9\end{array}$ \\
\hline
\end{tabular}

En revanche, des arrière-effets néfastes ont également été notés. Nous distinguerons et discuterons successivement les effets transitoires et ceux qui se maintiennent stables après bouturage.

\section{A. Les effets transitoires de la vitroculture}

Lorsqu'ils se manifestent, les inconvénients majeurs des vitroplants sont liés à une poursuite de la prolifération végétative hors tube. La ramification précoce excessive produit des touffes de feuilles juvéniles dont le bilan nutritionnel est beaucoup moins favorable que celui d'une plante ne présentant qu'un seul méristème actif, donc un système foliaire bien agencé et plus efficace, à LAI (Leaf Area Index = rapport de la surface projetée à la surface développée du système foliaire) nettement réduit. Ce simple handicap trophique peut expliquer les retards de floraison, la diminution de la qualité florale et notamment du poids, des avortements précoces se traduisant par une production réduite et secondairement une aggravation de l'exubérance foliaire.

L'essai 2 indique clairement qu'une modification des techniques de vitroculture même minime, telle que la prolongation du dernier cycle de prolifération, peut améliorer sérieusement la restauration d'une dominance apicale stable de l'ensemble des variétés observées. Il paraît donc raisonnable de chercher une amélioration des techniques de vitroculture.

Compte tenu des exigences contradictoires d'une prolifération efficace (conditionnant les coûts de production) et d'une fourniture de plants non ramifiés, il paraît indispensable, en vue de préparer la sortie des vitroplants, de rompre la monotonie des cycles de prolifération pour réorienter la physiologie des explants et induire une activité méristématique attractive et mobilisatrice des métabolites disponibles. Cette rupture dans la conduite routinière de la phase de multiplication peut être de nature variable; pour des raisons pratiques, nous envisageons des modifications des conditions physiques (rythme de repiquage, conditions thermiques ou lumineuses...). La mise en œuvre d'un milieu de transition pauvre ou complètement dépourvu de substances de croissance mériterait également d'être expérimentée pour améliorer la qualité des vitroplants à leur sortie de tube.
La prolongation du dernier cycle de prolifération pendant 8 semaines avant la phase d'enracinement (traitement $\mathrm{V}$ de l'essai 2) améliore la qualité agronomique des vitroplants. Ce peut être l'effet de l'épuisement progressif général de ce milieu de transition mais aussi d'une probable évolution profonde des équilibres de base liée, d'une part, à une absorption préférentielle de certains éléments (peut être les cytokinines sont elles utilisées plus rapidement que l'auxine, l'azote serait assimilé plus vite que la potasse...), d'autre part, à la dégradation des vitamines et de certaines substances de croissance. Ces modifications réalisent des conditions favorables à la restauration de la dominance apicale et à la rhizogenèse de sorte que le maintien encore prolongé des explants dans ce milieu aboutit à leur enracinement spontané.

Par ailleurs, tous les essais réalisés traduisent une grande diversité variétale de comportement des vitroplants aussi bien en cours de vitroculture qu'en serre après sortie de tube. Cette observation suggère un ajustement concomitant du matériel végétal et des techniques de culture in vitro. Dans l'état actuel de nos connaissances, les améliorations des techniques de prolifération in vitro paraissent beaucoup plus limitées que les possibilités de sélection. Aussi exerçons-nous une sélection a posteriori pour l'aptitude à la vitroculture dans le cadre restreint de la technique de MAİA expérimentée à Fréjus. Toutefois, la précocité de floraison et le taux de ramification des génotypes bouturés paraissent être de bons critères de choix dans la mesure où ils sont corrélés avec la ramification précoce des vitroplants et ont une forte héritabilité. Les meilleurs génotypes auraient un profil moyen, les très précoces ne proliférant pas suffisamment in vitro et parmi les tardifs, seuls seraient acceptables ceux qui présentent des ramifications bien individualisées capables d'affirmer une forte dominance apicale stable lors de l'enracinement.

\section{B. Les effets stables de la vitroculture}

Deux clones, «Fredaisy » (tabl. 6) et «Fredimo» (tabl. 5), manifestent après vitroculture des caractéristiques nouvelles qui se maintiennent même après un cycle de bouturage; nous parlerons dans ce cas de multiplication non conforme.

De tels phénomènes, fréquemment signalés dans la littérature (MOUSSEAU, 1971 ; SIBI, 1971 ; ORTON, 1980...), surviennent généralement après régénération de cals et expriment soit des variations chromosomiques, soit des mutations géniques, soit des perturbations stables et héréditaires du contrôle épigénique.

Il sera très difficile d'avancer ici une interprétation indiscutable dans la mesure où nous ne pouvons pas faire l'analyse génétique de ce matériel hautement hétérozygote. Toutefois, il est possible que les 2 « déviants » ne résultent pas du même processus.

Dans le cas de «Fredimo», les 60 premiers vitroplants observés en 1977 étaient très comparables aux boutures traditionnelles, sauf 2 d'entre eux qui préfiguraient probablement l'ensemble du matériel in vitro fourni ultérieurement. On peut admettre qu'au cours des premiers cycles de multiplication in vitro, un événement (mutation?), apparu de novo ou préexistant (déchimérisation d'un tissu muté ayant d'ailleurs pu apparaître lors de la phase d'initiation du premier embryoïde), s'est manifesté de façon très nette chez 2 plants sur 60 ; la généralisation à l'ensemble du matériel diffusé par la suite proviendrait d'une simple dérive (entretien de la souche à partir d'un seul explant muté) ou résulterait d'un énorme avantage sélectif in vitro. 
Dans le cas de «Fredaisy » en revanche, nous pouvons envisager l'origine de la variation dès la formation du premier explant puisqu'aucun vitroplant conforme n'a jamais été observé.

Les 2 déviations stables observées sont donc apparues soit au cours, soit immédiatement après la phase d'induction de l'embryoïde initial différencié sur un jeune bouton floral. Cette différenciation se produirait, selon BECKER-ZENS (1981), en marge d'un petit cal à évolution très lente.

Toutefois, nous devons bien remarquer que ces 2 seuls cas concernent les 2 premiers clones de gerbera que MAÏA a mis en culture in vitro et qui lui ont servi de " cobayes" pour la mise au point des techniques culturales. Depuis ces premières tentatives, non seulement les vitroplants «Fredaisy 》 et "Fredimo" n'ont plus dévié mais surtout les nombreux clones nouveaux multipliés in vitro (31 génotypes différents) se sont avérés conformes, selon nos capacités d'appréciation. Les techniques employées paraissent donc aujourd'hui très fiables; néanmoins, par précaution :

- Tous les clones nouveaux multipliés in vitro font l'objet d'un test de conformité ; le plut tôt possible nous choisissons des vitroplants racinés non ramifiés pour les comparer aux boutures traditionnelles.

- Dans le cadre du conservatoire variétal, pour éviter ou contrôler toute dérive éventuelle en cours de multiplication, nous entretenons pour chaque variété plusieurs lignées clonales séparément sur un milieu minimum en substances de croissance.

- Lorsque la mise en culture axénique deviendra une opération banale pour le gerbera, il sera recommandé d'alterner la culture in vitro et un passage en conditions naturelles.

\section{CONCLUSION}

La multiplication in vitro ouvre des perspectives nouvelles à la fois aux sélectionneurs, aux multiplicateurs et aux utilisateurs du gerbera.

- Les nouveautés variétales peuvent être rapidement diffusées ; c'est un avantage considérable pour faire face à une situation mouvante (technique et commerciale), mais c'est également un risque de prolifération pléthorique de variétés mal connues.

- Le matériel végétal fourni à l'horticulteur peut présenter des qualités agronomiques très supérieures à condition d'appliquer aux cultivars aptes des règles de vitroculture correctes. D'importants progrès sont à attendre des efforts combinés de la sélection et du perfectionnement des techniques de culture in vitro.

Reçu le 22 décembre 1982. Accepté le 29 avril 1983.

\section{RÉFÉRENCES RIBLIOGRAPHIQUES}

Athanassiou I., 1979. Contribution à l'étude du pouvoir pathogène chez le couple Phytophthora cryptogea - Gerbera. Thèse Docteur Ingénieur, Orsay 1979, $77 \mathrm{p}$.

Becker-Zens R., Andrea-Zens, 1981. In vitro regencration in Pelargonium and Gerbera cultivars. Congrès SIHS, Hambourg, 1981.

Duclos A., Meynet J., 1977. Etude comparative de clones produits par bouturages et d'hybrides $\mathrm{F} 1$ de semis de gerbera. Travaux sur le Gerbera ; Journée d'étude du 16 novembre 1977, Fréjus, publication I.T.I.H.

Maïa E., 1977. Propagation de clones de Gerbera jamesonii par culture in vitro. Travaux sur le Gerbera, journée d'étude du 16 novembre 1977, Fréjus, publication I.T.I.H.

Mousseau J., 1971. Fluctuation induite par la néoformation de bourgeons in vitro. Coll. Culture des tissus de plantes, Strasbourg, 1970, 293, 235-239.
Murashige T., Serpa M., Jones J. B., 1974. Clonal multiplication of Gerbera through tissue culture. Hortic. Sci., 9 (3), 175-180.

Orton T. J., 1980. Chromosomal variability in tissue cultures and regenerated plants of Hordeum. Theor. appl. Genet., 56, 101-112.

Pierik R. M. L., Steegmans H. H. M., Marchis J. J., 1973. Gerbera plantlets from in vitro cultivated capitulum explants. Sci Hortic., 1 (1), 117-119.

Pierik R. M. L., Jansen J. L. M., Maasdam A. M., Binnendijk C. M., 1975. Optimalization of gerbera plantlet production from excised capitulum explants. Sci. Hortic., 3, 351-357.

Sibi M., 1971. Création de variabilité par culture de tissus in vitro chez Lactuca sativa. DEA Amel. Plantes; Orsay, $51 \mathrm{p}$. 